\title{
Nondisjunction and transmission ratio distortion of Chromosome 2 in a (2.8) Robertsonian translocation mouse strain
}

\author{
Reiner Schulz, ${ }^{1}$ Lara A. Underkoffler, ${ }^{2}$ Joelle N. Collins, ${ }^{2}$ Rebecca J. Oakey ${ }^{1}$ \\ ${ }^{1}$ Department of Medical and Molecular Genetics, King's College London, 8th Floor Guy's Tower, London, SE1 9RT, United Kingdom \\ ${ }^{2}$ Division of Human Genetics, The Children's Hospital of Philadelphia, Philadelphia, Pennsylvania 19104, USA
}

Received: 19 September 2005 / Accepted: 25 November 2005

\begin{abstract}
Aneuploidy results from nondisjunction of chromosomes in meiosis and is the leading cause of developmental disabilities and mental retardation in humans. Therefore, understanding aspects of chromosome segregation in a genetic model is of value. Mice heterozygous for a (2.8) Robertsonian translocation were intercrossed with chromosomally normal mice and Chromosome 2 was genotyped for number and parental origin in 836 individuals at $8.5 \mathrm{dpc}$. The frequency of nondisjunction of this Robertsonian chromosome is $1.58 \%$. Trisomy of Chromosome 2 with two maternally derived chromosomes is the most developmentally successful aneuploid karyotype at $8.5 \mathrm{dpc}$. Trisomy of Chromosome 2 with two paternally derived chromosomes is developmentally delayed and less frequent than the converse. Individuals with maternal or paternal uniparental disomy of Chromosome 2 were not detected at $8.5 \mathrm{dpc}$. Nondisjunction events were distributed randomly across litters, i.e., no evidence for clustering was found. Transmission ratio distortion is frequently observed in Robertsonian chromosomes and a bias against the transmission of the (2.8) Chromosome was detected. Interestingly, this was observed for female and male transmitting parents.
\end{abstract}

\section{Introduction}

Aneuploidy results from nondisjunction of chromosomes (Chrs) in meiosis. In humans, an estimated $10 \%-30 \%$ of fertilized eggs are trisomic or monoso-

Correspondence to: Rebecca J. Oakey; E-mail: rebecca.oakey@ genetics.kcl.ac.uk mic, which has profound clinical consequences (Hassold and Hunt 2001). Aneuploidy is the leading cause of developmental disabilities and mental retardation (Hassold and Hunt 2001) and occurs in approximately $5 \%$ of clinically recognized pregnancies (Warburton 1997) and in $0.3 \%$ of newborns (Hassold 1985). The overall incidence of aneuploidy (trisomy or monosomy) among fertilized mouse eggs is around 1\%-2\% (Bond and Chandley 1983). All mouse trisomies are retarded in growth and development. Most are lethal before 16 days postcoitum (dpc) and very few embryos survive to birth. Only trisomy 19 mice have been observed postnatally. Autosomal monosomies are generally not viable (Hernandez and Fisher 1999).

Robertsonian $(\mathrm{Rb})$ Chrs in mice are the result of a centric fusion of two Chrs forming a single metacentric Chr without an apparent loss of genetic material and with no obvious phenotypic abnormality, although minor structural differences may arise during the formation of some $\mathrm{Rb}$ Chrs (Davisson and Akeson 1993). Rb Chrs occur naturally in some mouse populations, and although these mice have $39 \mathrm{Chrs}$, in the case of a single Rb-carrying strain they have $40 \mathrm{Chr}$ arms and, thus, a complete diploid set. However, mice heterozygous for $\mathrm{Rb}$ translocations undergo an increased rate of nondisjunction compared to karyotypically normal mice (Beechey and Evans 1996; Cattanach et al. 2004) and provide a model for studying $\mathrm{Chr}$ segregation. In this study, (2.8) Rb translocation-carrying mice were intercrossed with mice that do not carry this translocation and the incidence of nondisjunction of $\mathrm{Chr} 2$ was determined, the phenotypes of aneuploid embryos were considered, and the inheritance pattern of this $\mathrm{Rb}$ Chr was investigated.

The mouse is a good model in which to study the transmission of $\mathrm{Rb}$ Chrs because these anomalies are frequently maintained on defined genetic back- 
grounds, and the parent-of-origin of specific Chrs can be determined by several methods including molecular genotyping of polymorphic genetic markers. Genotyping can be performed at any stage during development and is not dependent on the presence of a phenotypic marker on the Chr of interest. Wild populations of $\mathrm{Rb}$ translocation mice exist mostly in Western Europe and North Africa (Redi and Capanna 1988). The heterozygotes of these populations show relatively low frequencies of spontaneous nondisjunction, as do karyotypically normal laboratory mouse strains (Beechey and Evans 1996). However, as wild $\mathrm{Rb}$ derivatives were introduced into laboratory mouse genetic backgrounds, significant increases in the frequency of nondisjunction were observed (Wallace et al. 1992). Thus, the incidence of nondisjunction can be studied between different strains of mice harboring $\mathrm{Rb}$ Chrs. The strain used here carries a (7.18) and a (2.8) Rb Chr. The (7.8) Rb Chr has been studied elsewhere (Oakey et al. 1995; Underkoffler et al. 2002). Here, the focus is on the (2.8) Rb Chr, and the frequency of nondisjunction is compared with the frequency for the (7.18) Rb Chr.

Transmission ratio distortion (TRD) is the unequal representation of alleles or Chrs (PardoManuel de Villena and Sapienza 2001). Such deviations from a 1:1 Mendelian transmission ratio have been reported in human and mouse, but the mechanisms involved are not completely understood. TRD is often associated with $\mathrm{Chr}$ rearrangements including $\mathrm{Rb}$ Chrs (Aranha and Martin-DeLeon 1991; Gropp and Winking 1981). Several regions of the genome have been associated with TRD, including the $\mathrm{Om}$ locus on mouse $\mathrm{Chr}$ 11 (Pardo-Manuel de Villena et al. 2000). TRD of the (7.18) $\mathrm{Rb}$ Chr has been reported previously (Underkoffler et al. 2005), and now the (2.8) Rb Chr is examined in a similar cross.

\section{Materials and methods}

Mice. All mouse strains were obtained from The Jackson Laboratory. The $\mathrm{Rb}(2.8) 2 \mathrm{Lub}(7.18) 9 \mathrm{Lub}$ or $\mathrm{Rb9}(2.8) 2 \mathrm{Lub}(\mathrm{R})$ and C57BL/6JEi-Rb/7.18)9Lub (B) strains were obtained from the Cytogenetic Models Resource at The Jackson Laboratory. The R strain has been maintained on its own inbred background, a combination of approximately 50\% wild-derived Mus musculus domesticus and 50\% laboratory mouse strain background (Davisson and Akeson 1993). The B strain was derived by repeated backcrossing to a C57BL/6J-Ei background. (2.8) Rb Chr occurs only in the $\mathrm{R}$ strain of mice and is heterozygous in one parent at the $F_{1}$ breeding stage. $R$ mice were crossed to $\mathrm{DBA} / 2 \mathrm{~J}$ (D) to generate $\mathrm{F}_{1}$ progeny heterozygous for the $(2.8) \mathrm{Rb} \mathrm{Chr}$. B mice were crossed either to $\mathrm{C} 3 \mathrm{H} / \mathrm{HeJ}$ (C3) (producing 518 $\mathrm{F}_{2}$ individuals) or to Mus musculus castaneus (cast) (producing $318 \mathrm{~F}_{2}$ individuals) and carried no (2.8) $\mathrm{Rb}$ Chr. Mus musculus castaneus and $\mathrm{C} 3 \mathrm{H} / \mathrm{HeJ}$ strains were both used because C57BL/6JEi-Rb/7.18)9Lub $\times$ Mus musculus castaneus $\mathrm{F}_{1}$ males are sterile and, in the first instance, we wanted to observe reciprocal crosses. (2.8) Rb heterozygotes were crossed to mice without a $(2.8) \mathrm{Rb}$ Chr to produce $\mathrm{F}_{2}$ progeny harvested at $8.5 \mathrm{dpc}$ (Oakey et al. 1995). Eight hundred thirty-six $F_{2}$ embryos were genotyped for number and parental origin of Chr 2. For a subset of 631 embryos from 128 litters, the genotypes of all littermates were determined and these embryos were used to investigate clustering of nondisjunction events among litters. For TRD studies, the frequencies of embryos with a balanced karyotype only were investigated (816 embryos).

DNA preparation. A small tissue sample was dissected from the tail region of each embryo and frozen for DNA preparation. DNA was isolated as in Oakey et al. (1995).

Genotyping. The simple sequence repeat (SSR) polymorphism primer D2MIT32 (which maps at around $11 \mathrm{cM}$ on $\mathrm{Chr} 2$ ) was used in conventional radioactive PCR genotyping (Underkoffler et al. 2002) for 330 cast strain-containing DNA samples. The allele sizes for D2MIT32 were B, 86 bp; R, 100 bp; D, $102 \mathrm{bp}$; and cast, $84 \mathrm{bp}$. D2MIT442 was used for the remaining samples (mostly $\mathrm{C} 3 \mathrm{H} / \mathrm{HeJ}$ containing) in an automated genotyping assay with fluorescently labeled primers. The allele sizes for D2MIT442 (mapping at around $50 \mathrm{cM}$ on $\mathrm{Chr} 2$ ) with fluorescently labeled primers are as follows: B, 123 bp; R, 120 bp; D, 136 bp; C3, 105 bp; and cast, 115 bp. The fluorescent signal from the PCR products was read on an ABI 3730 DNA analyzer and the size of the products was scored using the ABI GeneMapper 3.0 (Applied Biosystems, Foster City, CA). Two independent individuals scored all of the genotypes (on genotyping gels or on ABI size readout). The embryo genotypes were scored for number of Chr 2's (normal or trisomic), and the parental and grandparental origin was determined for the Chr 2's. For a small subset of samples (10), the genotypes were not equivocally scored with these two markers because of low DNA quality; these were further confirmed using D2MIT493 (around $72 \mathrm{cM}$ on $\mathrm{Chr}$ 2) allele sizes B, $112 \mathrm{bp}$; R, $110 \mathrm{bp}$; D, $98 \mathrm{bp}$; C3, $125 \mathrm{bp}$; and cast, $120 \mathrm{bp}$; D2MIT456 (around $86 \mathrm{cM}$ on Chr 2) with allele sizes of approximately B, 105 bp; R, 108 bp; D, $170 \mathrm{bp}$; C3, $180 \mathrm{bp}$; and cast, $200 \mathrm{bp}$ or 
D2MIT395 (around $68 \mathrm{cM}$ on Chr 2) with allele sizes of $\mathrm{B}, 128 \mathrm{bp}$; R, $120 \mathrm{bp}$; D,134 bp; C3, $122 \mathrm{bp}$; and cast, $136 \mathrm{bp}$.

Data acquisition. Data from $836 \mathrm{Chr} 2$ genotypes ( 210 litters) were assessed for normal $\mathrm{Chr}$ disjunction, trisomy, and uniparental disomy (UpDi). Eight hundred sixteen genotyped embryos with a balanced chromosomal complement were also characterized with respect to the parental and grandparental strain, sex of the $\mathrm{Rb}$-transmitting grandparent and parent, and the embryonic phenotype (normal for the day of harvest or growth retarded). Chromosomally balanced embryos were scored as having no $\mathrm{Rb} \mathrm{Chr}$, only a maternally derived $\mathrm{Rb} \mathrm{Chr}$, or only a paternally derived $\mathrm{Rb}$ Chr.

\section{Statistical methods}

Clustering of nondisjunction events. For a subset of 128 litters (631 embryos), complete genotyping data for all recognizable embryos in a litter was available (not including reabsorptions). Under the null hypothesis of the absence of any clustering effect, i.e., under the assumption that nondisjunction events are uniformly randomly distributed among the embryos, one expects litters to contain $X=0,1$, $\geq 2$ nondisjunction events with certain absolute frequencies, given the total number of 20 nondisjunctions. These expected absolute frequencies were estimated via computer simulation that randomly distributed 20 nondisjunction events among 631 embryos where each embryo could attract at the most two nondisjunctions. This simulation was repeated 10,000 times, each time with a different initial random number generator seed. The numbers of litters with $X=0,1, \geq 2$ nondisjunctions, averaged over the 10,000 runs of the simulation, constitute the estimated expected absolute frequencies to which the actual observed frequencies were compared using a standard $\chi^{2}$ goodness-of-fit test.

A direct, per-litter $\chi^{2}$ test of the observed number of nondisjunction events versus the maximum likelihood estimate of the expected number (total number of nondisjunctions $=20 /$ total number of meioses $=1262$, times twice the number of embryos in the litter) was also performed. However, the very low $(<1)$ expected number of nondisjunction events per litter renders the result of this test questionable. The above computer simulation was therefore reused to estimate the test's true $p$ value in terms of the relative frequency with which the $\chi^{2}$ value for the simulated distribution of nondisjunction events among the litters was equal to or exceeded the $\chi^{2}$ value for the observed distribution.

Finally, the generalized likelihood ratio (GLR), which also follows the $\chi^{2}$ distribution, was computed for the two statistical models L0 and L1 that correspond to the null hypothesis of a fixed probability of nondisjunction that is the same for all litters and the alternative hypothesis of an individual probability per litter, respectively. In general, GLR compares the two models in terms of their ability (probability) to generate the observed data. Here, $\quad \mathrm{GLR}=-2 \log (\mathrm{LO} / \mathrm{L} 1)$, with $\mathrm{LO}=\Pi_{i} B i[a(i)$, $2 n(i), p]$ and $\mathrm{L} 1=\Pi_{i} B i[a(i), 2 n(i), p(i)]$, where $B i$ is the probability to observe $a(i)$ nondisjunction event in the litter $i$ with $n(i)$ embryos [2n(i) meioses] according to the binomial distribution and a litterindependent nondisjunction probability $p$ or a litterdependent probability $p(i) . p$ and $p(i)$ are the maximum likelihood estimates for the given data and the respective hypothesis, i.e., $p=20 / 1262$ and $p(i)=a(i) /[2 n(i)]$.

Transmission ratio distortion (TRD). Standard $\chi^{2}$ goodness-of-fit tests on $2 \times 2$ contingency tables were conducted to obtain the results on TRD in this cross.

\section{Results and discussion}

Incidence of nondisjunction. Nondisjunction in both mouse and human usually involves errors in meiosis where one round of DNA replication is followed by two cellular divisions to give haploid gametes. Meosis I (MI) separates homologous Chrs, meiosis II (MII) segregates sister chromatids of each homolog, and nondisjunction can occur at either stage (Lamb and Hassold 2004). In humans, there is evidence for Chr-specific affects that influence individual $\mathrm{Chr}$ nondisjunction. For example, Chr 16 trisomy is mostly a result of maternally derived errors in MI, and sex Chr anomalies and trisomy 18 are frequently associated with MII errors and MII nondisjunction, respectively (Hassold and Hunt 2001). Thus, it is important to study the behavior of individual chromosomes. Factors that can affect the frequency of nondisjunction include centromere position, the size of the Chr in question, and the position of repeat sequences (Warburton and Kinney 1996).

We determined the frequency of nondisjunction for mouse Chr 2 by counting trisomies and UpDi but not monosomies because these are not viable at 8.5 $\mathrm{dpc}$ in a cross with one parent being heterozygous for (2.8) $\mathrm{Rb}$ Chr. The number of observed nondisjunction events at $8.5 \mathrm{dpc}$ for Chr 2 was 20 
Table 1. Observed distribution of nondisjunction events for (2.8) $\mathrm{Rb}$ Chr and $\chi^{2}$ test results for the comparison with the expected distribution

\begin{tabular}{llllll}
\hline No. Nondisjunctions & 0 & 1 & 2,3 , or $>3$ & Total & $p(d f=2)$ \\
\hline No. litters observed & 109 & 18 & 1 & 128 & \\
No. litters expected & 109.5485 & 16.9850 & 1.4665 & 128 & 0.2118 \\
$\chi^{2}$ & 0.0027 & 0.0607 & 0.1484 & 0.8995 \\
\hline
\end{tabular}

The $p$ value (0.8995) is not significant. A subset of 631 embryos from 128 completely genotyped litters was used for this calculation (no partially genotyped litters were included).

Table 2. Frequencies of phenotypes for embryos with Chr 2 aneuploidy and parental origin of the extra Chr in the trisomies

\begin{tabular}{lll}
\hline Karyotype & Phenotype & Number \\
\hline Mat UPD 2 & Normal & 0 \\
Mat UPD 2 & Growth retarded & 0 \\
Pat UPD 2 & Normal & 0 \\
Pat UPD 2 & Growth retarded & 0 \\
Mat Trisomy & Normal & 9 \\
Mat Trisomy & Growth retarded & 8 \\
Pat Trisomy & Normal & 0 \\
Pat Trisomy & Growth retarded & 3 \\
\hline
\end{tabular}

$(1.58 \%)$. By comparison, the frequency of nondisjunction for a cross where both parents were heterozygous for (7.18) $\mathrm{Rb} \mathrm{Chr}$ combining both Chrs 7 and 18 was $15.9 \%$ (Underkoffler et al. 2002). The frequency of nondisjunction for an analogous cross involving (2.6) $\mathrm{Rb} \mathrm{Chr}$ and determined by karyotyping oocytes was reported to be $17 \%$ (Chewbotar and Barilyak 1994), and a double Rb heterozygote intercross was found to have a frequency of nondisjunction as high as $28 \%-36 \%$ (Beechey and Searle 1988). The reason for such a low frequency of nondisjunction compared with the $\mathrm{Rb}(7.18)$ cross is largely the result of (2.8) $\mathrm{Rb} \mathrm{Chr}$ being present in only one parent and because Chr 2 but not Chr 8 nondisjunctions were followed. In addition, it is also possible that nondisjunction is Chr-specific and Chr 2 experiences a low frequency of nondisjunction. This could be attributed to some aspect of $\mathrm{Chr}$ structure, e.g., lacking or having additional repeats, the size of the $\mathrm{Chr}$, or in the relative position or efficiency of the $\mathrm{Rb}$ centromere compared with that of other Rbs and to the acrocentric Chrs.

Clustering of nondisjunction events. Previous studies of a (7.18) Rb Chr heterozygous in both parents of a cross have shown that the distribution of nondisjunction events across litters is not random but that some litters contain more and other litters fewer than expected embryos that result from nondisjunction events compared with other individuals.
This may be the result of genetic background where some males or females were prone to a particularly high incidences of nondisjunction. We investigated this phenomenon for the (2.8) Rb Chr for whom the observed distribution of nondisjunction events is given in Table 1.

The observed distribution was compared with the distribution that is expected under the assumption of a uniform, litter-independent probability for a nondisjunction event to take place. A $\chi^{2}$ test, detailed in Table 1, found no difference between the observed and the expected distribution $\left(\chi^{2}=0.2118\right.$, $p=0.8995, d f=2)$, providing no evidence for a clustering effect, i.e., the probability of nondisjunction was litter-dependent. The theoretically expected distribution almost perfectly fit a Poisson distribution with $\lambda=0.1563\left(\chi^{2}=0.0033, p=0.9984\right.$, $d f=2$ ), and, consequently, so did the actual occurrence of nondisjunction events among mouse litters.

The test result was similar when we did not use summary statistics (number of litters with $\mathrm{X}$ nondisjunctions) but used the raw data (number of embryos and number of nondisjunction events per litter). A direct comparison of the observed number of nondisjunctions in a litter with the expected number yields $\chi^{2}=111.8399$ and $p=0.8290$ $(d f=127)$, which also does not lend support to the clustering hypothesis. In 10,000 runs of the computer simulation that was designed to estimate the true $p$ value of this test, the $\chi^{2}$ value for the simulated random distribution of nondisjunction events among the litters was equal to or exceeded $\chi^{2}=111.8399$ for the observed distribution 7888 times so that, with this method, $p=0.7888$, which is comparable to and consistent with the original $p$ value of 0.8290 . The GLR for the given data was 74.8295. Since GLR follows the $\chi^{2}$ distribution, $p=0.9999(d f=127)$. So, the GLR-based $\chi^{2}$ analysis also provided no evidence for clustering.

Taken together, the test results suggest no obvious skewing of nondisjunction events. (2.8) Rb Chr is present in only one of the parents of the cross, thus reducing the overall number of nondisjunction events per litter of mice. This reduction may have 
been responsible for the absence of measurable clustering, or (2.8) Rb Chr may not be as susceptible to skewing as the (7.18) Rb Chr.

Chromosomal aneuploidy and embryo development. The phenotypes and numbers of embryos identified with Chr 2 trisomy are listed in Table 2. As expected, autosomal monosomies are not detected at $8.5 \mathrm{dpc}$ in the mouse because monosomies, unlike trisomies, are not compatible with even early embryonic development and, thus, no information on this class of aneuploidy has been obtained in this study. No paternal UpDi (PatUpDi) or maternal UpDi (MatUpDi) individuals were identified in 836 embryos, which is as expected because a nondisjunction event has to take place in both the maternal and the paternal gametes and one of the parents in this cross does not carry a $\mathrm{Rb} \mathrm{Chr}$ and nondisjunction frequency is very low $(>0.1 \%)$ in chromosomally normal mice. Three trisomies with two paternal and one maternal Chr 2 were observed, all growth retarded. This karyotype is developmentally disadvantaged. A total of 17 trisomies with two maternal and one paternal Chr 2 were identified at $8.5 \mathrm{dpc}$, over half of which were developmentally normal. Embryos with Chr 2 trisomy involving two maternal Chr 2's appear to be developmentally more successful than embryos with two paternal Chr 2's. Since trisomy can result from nondisjunction in a single parent, trisomies were expected in this cross.

Of the 20 trisomies, four had an additional trisomy of Chr 7 and one had an additional trisomy of Chr 18 from the second $\mathrm{Rb} \mathrm{Chr}$ that segregates in this cross. Four of these had a normal phenotype for the day of harvest and one individual, which had trisomy of Chr 2 with an extra maternal Chr 2 plus Chr 7 trisomy, was retarded in development. Thus, the second trisomy did not retard the growth of the embryo in four of five cases at $8.5 \mathrm{dpc}$. In the one doubletrisomy (for 7 and 2) embryo with a retarded phenotype, either one or the other or a combination of both could have contributed to its developmental demise.

Effects of recombination on $\mathrm{Chr} 2$ will not have a profound effect on scoring the incidence of trisomic embryos. However, recombination may affect the identification of UpDi genotypes. When Chrs recombine, the parental origin of a given Chr may effectively be misidentified. A subset of Chrs with (2.8) Rb were typed with two or more markers at different loci on Chr 2 (proximal and distal) regions and a low frequency of recombination was detected (1/30 Chrs typed disparately at proximal and distal regions of the Chr, unpublished data). However, since UpDi would have been a very rare event in this cross, it is unlikely that any UpDis were misidentified.
Reciprocal translocations, including the $\mathrm{T} 28 \mathrm{H}$, $\mathrm{T} 26 \mathrm{H}, \mathrm{T} 11 \mathrm{H}, \mathrm{T} 1 \mathrm{Sn}$, and T2Wa strains (Beechey et al. 2004), have been used to generate uniparental duplications (UpDp) of mouse Chr 2. These chromosomal anomalies have been used to define phenotypes resulting from UpDp of proximal Chr 2 . Animals with two paternal copies from the $\mathrm{T} 11 \mathrm{H}$ breakpoint to the centromere have a placental overgrowth phenotype but are normal-sized viable fetuses. Two maternal copies of the same region result in small placentas and embryos and viable but small adults. In the other direction, two maternal copies of distal Chr 2 (between T2Wa and T26H) yield a lethargic/nonfeeding lethal (by day 1) phenotype, and two paternal copies result in an edematous, hyperactive, lethal (by day 4) phenotype. These phenotypes are caused by misexpression of imprinted genes residing in these regions. The map of imprinted phenotypes for the most part has been generated from reciprocal translocations that produce combinations of partial disomies and, thus, some of the phenotypes can result from interactions between imprinted loci on the different Chrs that participate in the translocation, in effect modifying the single Chr phenotype. A combination of imprinting effects has been shown to modify phenotypes on Chr 2 (Cattanach et al. 2004). For example, an embryonic lethal phenotype from MatDp proximal Chr 2 mice (Cattanach and Kirk 1985) recently has been attributed to interactions with imprinted genes on PatDp proximal Chr 11 (Cattanach et al. 2004).

Several well-characterized imprinted genes that reside on Chr 2 have been studied, notably, Gnas, GnasXL, Nesp (Peters et al. 1999), Nespas (Wroe et al. 2000) Nnat (Kagitani et al. 1997), and recently Gatm (Sandell et al. 2003). Possible imprinted genes not yet identified could include the mouse homologs of the WT1 antisense (WT1-AS) and alternative transcript (AWT1) genes that are both paternally expressed in human (Dallosso et al. 2003). Misexpression of imprinted genes could contribute to the embryonic demise of maternal and paternal UpDi mice before $8.5 \mathrm{dpc}$, but more likely these categories of potential offspring do not occur in this cross because the frequency of nondisjunction in non- $\mathrm{Rb}$ mice is very low.

Previous complementation studies on $\mathrm{Chr} 2$ with another $\mathrm{Rb}$ translocation, $\mathrm{Rb}$ (2.18) 6Rma (Beechey and Searle 1987), showed that no maternal or paternal disomic Chr 2 mice were born but one small PatUpDi embryo was seen at $14.5 \mathrm{dpc}$ from a total of 175 embryos. Specifically, reciprocal crosses of R6Rma/+ males or females with R6Rma $p a /+p a$ yielded the following offspring: with marked $(p a / p a)$ 
mothers, 0/137 MatUpDi (pa/pa) and 0/102 PatDi (+/ + ) were seen at birth. Also, no MatUpDi (pa/pa) embryos were seen in midgestation (0/160). With marked $(p a / p a)$ fathers, no PatUpDi $(p a / p a)$ mice were born (0/157) and no MatUpDi $(+/+)$ were born (0/147), but one PatUpDi (pa/pa) embryo of a total of 175 embryos examined was seen at $14.5 \mathrm{dpc}$. This embryo was smaller than its littermates (Beechey and Searle 1987).

Two-paternal Chr 2 trisomies are rare and growth retarded at $8.5 \mathrm{dpc}$, possibly partly because of misexpression of imprinted genes, but also because of gene dosage effects of the third Chr 2. The incidence of two-maternal $\mathrm{Chr} 2$ trisomies is higher than two-paternal Chr 2 trisomies, and over half of these embryos are normal at $8.5 \mathrm{dpc}$. Because the gene dosage of nonimprinted genes is likely to be equivalent in trisomies of maternal and paternal origin, the increased viability of this trisomy over the two-paternal type supports the hypothesis that two-paternally derived $\mathrm{Chr} 2$ 's cause a more severe phenotype than two-maternally derived Chr 2's at this developmental time point. Mice that are trisomic for a whole autosome are not normally viable (Hernandez and Fisher 1999), and gene dosage intolerance has devastating developmental consequences. In humans, trisomy 21 occurs as a result of chromosomal nondisjunction at a frequency of 1 in 800 live births (Patterson and Costa 2005). Two principal hypotheses exist for the formation of detrimental phenotypes as a result of Chr 21 trisomy. The gene dosage effect hypothesis states that elevated expression of specific genes leads to a phenotype. The amplified development instability hypothesis states that an elevated activity of sets of genes decreases genetic stability and results in a developmental phenotype (Patterson and Costa 2005). Both of these hypotheses could apply to the situation observed in mouse Chr 2 trisomy.

\section{Observed versus expected numbers of embryos} with Chr 2 trisomy. Figure 1 illustrates that under the assumption of an equal probability for each possible combination of gametes in a cross between a karyotypically normal mouse and a mouse that is heterozygous for the $(2.8) \mathrm{Rb} \mathrm{Chr}$, the relative frequencies of chromosomally balanced offspring, offspring trisomic for Chr 2, and offspring with uniparentally derived Chr 2 should be $0.4,0.4$, and 0.2 , respectively. However, for the set of $631 \mathrm{em}$ bryos for whom the genotypes of all littermates were known, the observed relative frequencies were $0.9683,0.0317$, and 0 (611 normal and 20 trisomic

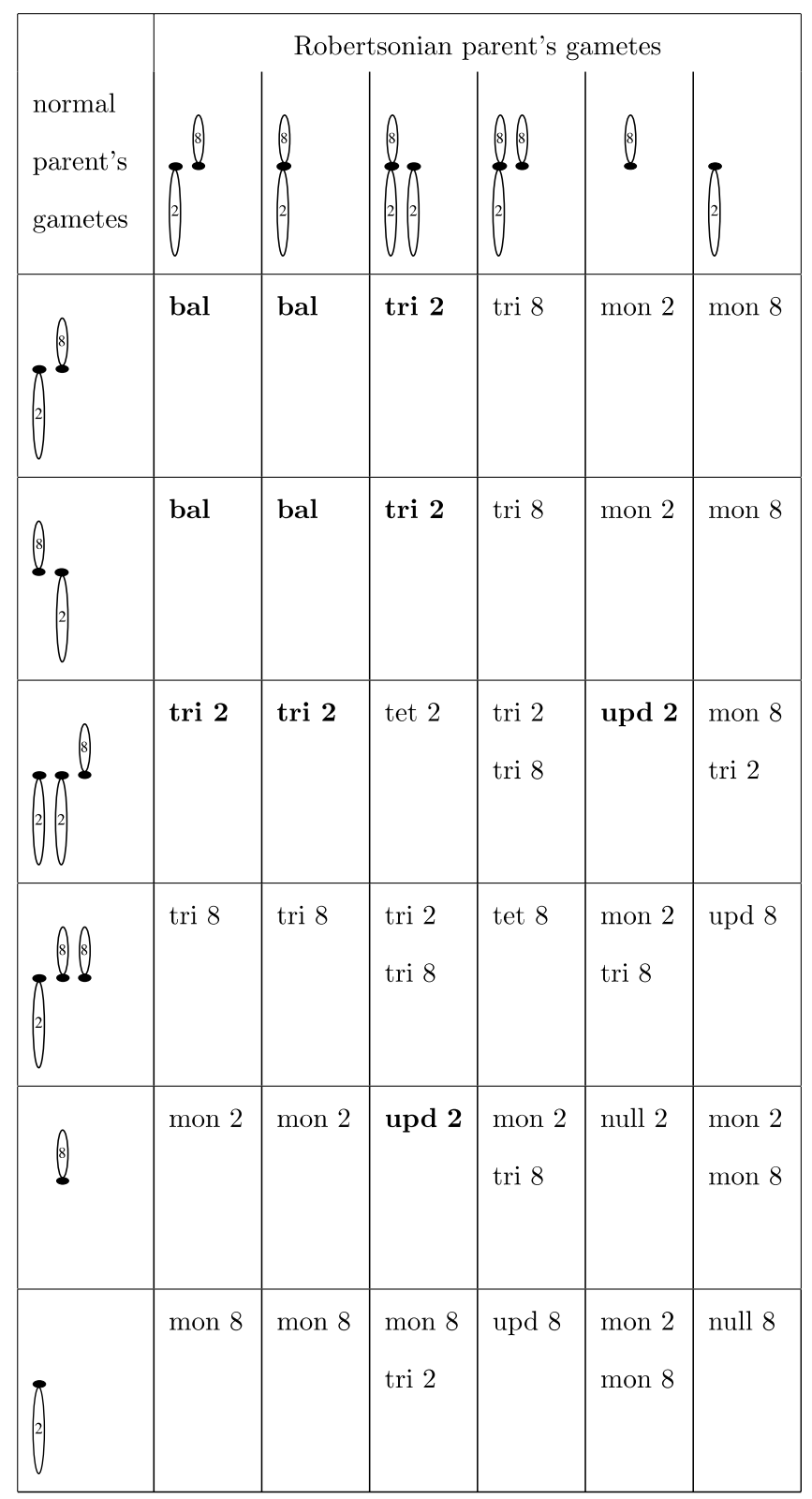

Fig. 1. The combinations of gametes that can arise in a mating of a normal mouse (rows) with a mouse that is heterozygous for the Robertsonian translocation of Chrs 2 and 8 (columns). Gametes that are diploid or nullisomic for both Chrs 2 and 8 are possible, but they are not shown here because they were not observed since they are subject to lethality before the day of harvest. The normal parents are most likely to produce one Chr 2 and one Chr 8 (first two rows) since nondisjunction is not elevated in a non- $\mathrm{Rb}$ mouse strain and, thus, combinations in rows 3-6 are extremely unlikely.

embryos). This can be explained by a lack of the disomic and nullisomic gametes (especially in the non- $\mathrm{Rb}$ parent) that are necessary to produce trisomic or UpDi offspring or by an increased lethality of such offspring before $8.5 \mathrm{dpc}$. 
Table 3. Frequencies of embryos with a balanced set of Chrs (816), carrying either Chrs 2 and 8 or (2.8) Rb Chr, and the parental and grandparental origin of the $(2.8) \mathrm{Rb} \mathrm{Chr}$ if present

\begin{tabular}{|c|c|c|c|c|c|c|}
\hline Cross & No. embryos & $M M$ & $M P$ & $P M$ & $P P$ & $O$ \\
\hline $\mathrm{BC} \times \mathrm{DR}$ & 85 & & & & 40 & 45 \\
\hline $\mathrm{CB} \times \mathrm{DR}$ & 147 & & & & 68 & 79 \\
\hline $\mathrm{BC} \times \mathrm{RD}$ & 74 & & & 31 & & 43 \\
\hline $\mathrm{CB} \times \mathrm{RD}$ & 109 & & & 42 & & 67 \\
\hline $\mathrm{DR} \times \mathrm{BC}$ & 54 & & 27 & & & 27 \\
\hline $\mathrm{DR} \times \mathrm{CB}$ & 162 & & 70 & & & 92 \\
\hline $\mathrm{RD} \times \mathrm{CB}$ & 67 & 30 & & & & 37 \\
\hline $\mathrm{RD} \times \mathrm{BC}$ & 118 & 54 & & & & 64 \\
\hline Total & 816 & 84 & 97 & 73 & 108 & 454 \\
\hline
\end{tabular}

For example, in the third row, of 74 embryos resulting from the $\mathrm{BC} \times \mathrm{RD}$ cross, 31 embryos inherited the $(2.8) \mathrm{Rb}$ Chr through their father and grandmother $(\mathrm{PM}=$ paternal parental and maternal grandparental inheritance), while 43 embryos did not inherit the (2.8) Rb Chr. $\mathrm{B}=\mathrm{C} 57 \mathrm{BL} / 6 \mathrm{~J}-\mathrm{EiRb} / 7.18 / 9 \mathrm{Lub} ; \mathrm{C}=$ Mus mus castaneus or $\mathrm{C} 3 \mathrm{H} / \mathrm{HeJ} \mathrm{R}=\mathrm{Rb}(2.8) 2 \mathrm{Lub} / 7.18) 9 \mathrm{Lub} ; \mathrm{D}=\mathrm{DBA} / 2 \mathrm{~J}$. RD indicates a mouse derived from a $\mathrm{Rb}(2.8) 2 \mathrm{Lub} / 7.18$ ) $9 \mathrm{Lub}$ mother and a $\mathrm{DBA} / 2 \mathrm{~J}$ father which follows for all of the two-letter abbreviations. A RD $\times \mathrm{BC}$ cross is an RD mother crossed with a BC father.

Transmission ratio distortion. Transmission ratio distortion (TRD) is defined as a significant departure from the normally expected Mendelian ratios of inheritance of an allele or $\mathrm{Chr}$ (Pardo-Manuel de Villena and Sapienza 2001). TRD is observed among specific regions of the mouse and human genome and is frequently associated with $\mathrm{Chr}$ rearrangements such as Rbs. Structural changes like the loss of satellite sequences that occur as a result of the formation of $\mathrm{Rb}$ Chrs may affect gametic segregation of the homologous Chrs that partake in the $\mathrm{Rb}$ (Garagna et al. 2001; Ruvinskii et al. 1986). We wished to examine (2.8) Rb Chr for TRD. TRD is thought to result from the nonrandom segregation of Chrs during female meiosis (Pardo-Manuel de Villena and Sapienza 2001) where differences in meiotic spindle preferences affect the capture of centromeres by the egg and the polar body. It is also hypothesized that imprinting errors account for allele preference in certain regions of the genome (Croteau et al. 2003), including the imprinted Meg3/ Gt12-Dlk1 region of mouse Chr 12. Females in the cross described here transmit (7.18) $\mathrm{Rb} \mathrm{Chr}$ to their offspring less often than expected, but surprisingly heterozygous males transmit (7.18) Rb Chr significantly more frequently than the acrocentric Chrs 7 and 18 (Underkoffler et al. 2005). Nondisjunction of Chrs, for which imprinting effects are established, may be complicated by these genes. There could be increased losses of trisomic embryos compared with those involving nonimprinted Chrs, leading to an underestimation of nondisjunction and parent-oforigin skewing. Increased losses of trisomy 2 are unlikely since the phenotype of the distal Chr 2 imprinting effect at least is viable at $8.5 \mathrm{dpc}$. TRD is a phenomenon that is influenced by differences in gameteogenesis, but any effect on progeny outcome including imprinting is noteworthy.
The data for the (2.8) Rb Chr are summarized in Table 3. Theoretically, in a cross between a mouse that is heterozygous for the (2.8) Rb Chr and a mouse with a normal set of Chrs, the number of Rb-carrying chromosomally balanced offspring should equal the number of offspring with a normal and balanced set of Chrs. However, a strong bias against the transmission of the (2.8) $\mathrm{Rb} \mathrm{Chr}$ was observed, independent of the sex of the Rb-transmitting parent $\left(\chi^{2}=10.3725, p=0.0013, d f=1\right)$. This is generally consistent with findings in the mouse of a bias against transmission of $\mathrm{Rb}$ Chrs (Gropp and Winking 1981). In contrast, when the sex of the transmitting parent was taken into account, the results differed between male and female transmission and also compared with (7.18) $\mathrm{Rb} \mathrm{Chr}$ findings (Table 4). There was still clear evidence for a bias against the (2.8) $\mathrm{Rb} \mathrm{Chr}$ when it was carried by the father $\left(\chi^{2}=6.7687, p=0.0093\right)$ but not when the mother was the carrier $\left(\chi^{2}=3.7930, p=0.0515\right)$ (Table 4). In the case of (7.18) Rb Chr, male carriers had shown a significant bias in favor of its transmission, which had been an unexpected finding that was not observed with (2.8) Rb Chr. In females, the observed reduction in the bias against the transmission of the $\mathrm{Rb} \mathrm{Chr}$ when comparing the $(2.8)$ to the $(7.18) \mathrm{Rb}$ Chr was not significant $\left(\chi^{2}=0.0536, \mathrm{p}=0.8169\right)$ (Table 4). This observation is consistent with other studies that found reduced female transmission of $\mathrm{Rb}$ Chrs (Aranha and Martin-DeLeon 1991; Gropp and Winking 1981; Tease and Fisher 1991). Because with the (2.8) Rb Chr there was some evidence for its suppression in both females and males, the figures for females and males were compared directly and found to not differ significantly $\left(\chi^{2}=0.1349\right.$, $p=0.7135$ ). Finally, a test of whether the sex of the transmitting grandparent had any effect on the frequency of transmission did not uncover evidence in 
Table 4. Summary of $\chi^{2}$ test results

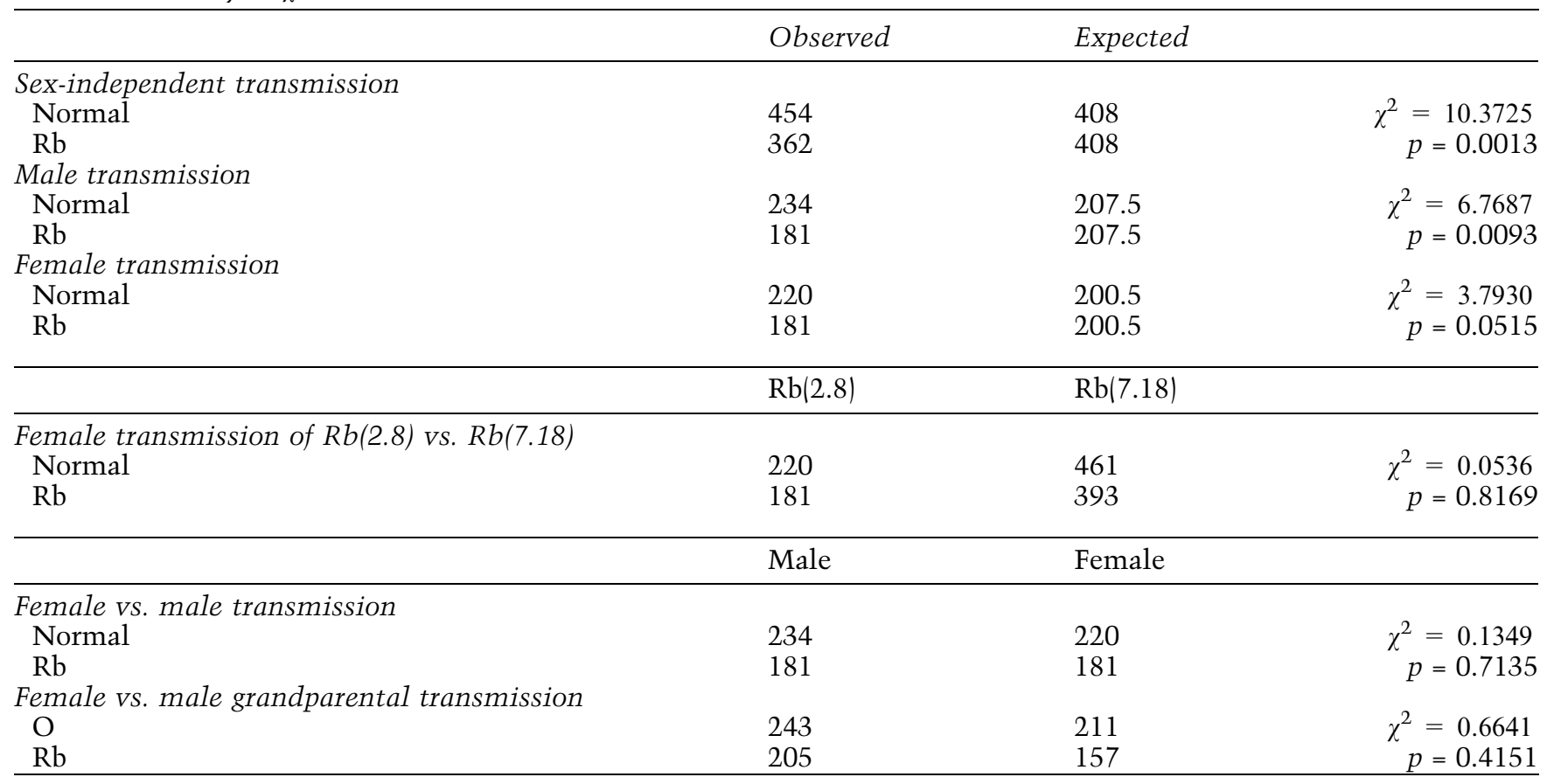

favor of that hypothesis $\left(\chi^{2}=0.6641, p=0.4151\right)$ (Table 4).

In humans, the causes of nondisjunction are not fully understood. MI errors are thought to predominate because the first division in females starts prenatally and is not completed until the time of ovulation. This lengthy process is likely to be errorprone, probably contributing to the relatively high incidence of aneuploidy in humans. One factor that is known to be associated with nondisjunction frequency is altered recombination, because recombinational exchange locks homologs together to promote correct segregation of Chrs (Lamb and Hassold 2004). Characterization of genetic factors associated with correct $\mathrm{Chr}$ segregation in mouse and human will help in understanding this process. The mouse is a good model to study the mechanisms of Chr segregation and then to correlate the findings with those in human. The developmental demise of aneuploid embryos will be elucidated by the identification of dosage-sensitive genes. This may be achieved in part by using existing models, like trisomy 16 mouse strains that model trisomy 21 in humans, but also by recent advances in chromosomal engineering that can generate precisely defined autosomal trisomies for the identification of dosage-sensitive genes that are responsible for developmental demise (Hernandez and Fisher 1999). These models will also be able to contribute to the resolution of the conflicting hypotheses about gene dosage versus the instability of sets of genes accounting for embryonic demise.

\section{Acknowledgments}

This work was supported by The Wellcome Trust (RJO), the Biotechnology and Biological Sciences Research Council (RS), and Public Health Service Grant number GM58759 from the National Institutes of Health (RJO). The authors thank Dr. Sheila Fisher for advice on statistical methods, Colin Beechey for advice on Robertsonian and reciprocal translocations, and Drs. Trevelyan Menheniott and Kathryn Woodfine for their critical reading of the manuscript.

\section{References}

1. Aranha IP, Martin-DeLeon PA (1991) The murine $\mathrm{Rb} / 6.16)$ translocation: evidence for sperm selection and a modulating effect of aging. Hum Genet 87, $278-284$

2. Beechey CV, Evans EP (1996) Numerical variants and structural rearrangements In: Lyon MF, Rastan S, Brown SDM (eds.) Genetic variants and strains of the laboratory mouse (Oxford: Oxford University Press), pp 1452-1505

3. Beechey CV, Searle AG (1987) Complementation studies on Chr2 with $\mathrm{Rb}(2.18) 6 \mathrm{Rma}$. Mouse Newslett 77,127 
4. Beechey CV, Searle AG (1988) Effects of zero to four copies of chromosome 15 on mouse embryonic development. Cytogenet Cell Genet 47, 66-71

5. Beechey CV, Cattanach BM, Blake A, Peters J (2004) Mouse imprinting map. Available at http://www. mgu.har.mrc.ac.uk/research/imprinting [accessed 05/ 01/06]

6. Bond DJ, Chandley AC (1983) The origins and causes of aneuploidy in experimental organisms In: Bond DJ, Chandley AC (eds.) Aneuploidy (Oxford: Oxford University Press), pp 27-54

7. Cattanach BM, Kirk M (1985) Differential activity of maternally and paternally derived chromosome regions in mice. Nature 315, 496-498

8. Cattanach BM, Beechey CV, Peters J (2004) Interactions between imprinting effects in the mouse. Genetics 168, 397-413

9. Chewbotar NA, Barilyak IR (1994) Comparison of the features of chromosomal nondisjunction during the first division of oocyte maturation in mice which are heterozygous for the balanced structural chromosomal rearrangements. Tsitol Genet 28, 11-19

10. Croteau S, Charron M-C, Latham KE, Naumova A K (2003) Alternative splicing and imprinting control of the Meg3/Gtl2-Dlk locus in mouse embryos. Mamm Genome 14, 231-241

11. Dallosso AR, Hancock AL, Brown KW, Williams AC, Jackson S, et al. (2003) Genomic imprinting at the WT1 gene involves a novel coding transcript (AWT1) that shows deregulation in Wilms' tumours. Hum Mol Genet 13(4), 405-415

12. Davisson MT, Akeson EC (1993) Recombination suppression by heterozygous Robertsonian chromosomes in the mouse. Genetics 133, 649-667

13. Garagna S, Marziliano N, Zuccotti M, Searle JB, Capanna E, et al. (2001) Pericentromeric organization at the fusion point of mouse Robertsonian translocation chromosomes. Proc Natl Acad Sci 98(1), 171-175

14. Gropp A, Winking H (1981) Robertsonian translocations: cytology, meiosis, segregation pattern and biological consequences of heterozygosity In: Berry RJ (ed.) Biology of the House Mouse (New York: Academic Press), pp 141-181

15. Hassold TJ (1985) The origin of aneuploidy in humans In: Dellarco VL, Voytek PE, Hollaender A (eds.) Aneuploidy (New York: Plenum Press), pp 103-115

16. Hassold TJ, Hunt P (2001) To err (meiotically) is human: the genesis of human aneuploidy. Nat Rev Genet 2, 280-291

17. Hernandez D, Fisher EMC (1999) Mouse autosomal trisomy. Trends Genet 15(6), 241-247

18. Kagitani F, Kuroiwa Y, Wakana S, Shiroishi T, Miyoshi N, et al. (1997) Peg5/Neuronatin is an imprinted gene located on sub-distal chromosome 2 in the mouse. Nucleic Acids Res 25(17), 3428-3432

19. Lamb NE, Hassold TJ (2004) Nondisjunction-A view from ringside. N Engl J Med 351(19), 1931-1934

20. Oakey RJ, Matteson PG, Litwin S, Tilghman SM, Nussbaum RL (1995) Nondisjunction rates and abnormal embryonic development in a mouse cross between heterozygotes carrying a $(7,18)$ Robertsonian translocation chromosome. Genetics 141, 667-674

21. Pardo-Manuel de Villena F, Sapienza C (2001) Female meiosis drives karyotypic evolution in mammals. Genetics 159, 1179-1189

22. Pardo-Manuel de Villena F, de la Casa-Esperon E, Williams JW, Malette J-M, Rosa M, et al. (2000) Heritability of the maternal meiotic drive system linked to $\mathrm{Om}$ and high-resolution mapping of the Responder locus in mouse. Genetics 155, 283-289

23. Patterson D, Costa ACS (2005) Down Syndrome and genetics-a case of linked histories. Nat Rev Genet 6, $137-147$

24. Peters J, Wroe SF, Wells CA, Miller HJ, Bodle D, et al. (1999) A cluster of oppositely imprinted transcripts at the Gnas locus in the distal imprinting region of mouse chromosome 2. Proc Natl Acad Sci 96(7), 3830-3835

25. Redi CA, Capanna E (1988) Robertsonian heterozygotes in the house mouse and the fate of their germ cells In: Daniel A (ed.) The cytogenetics of mammalian autosomal rearrangements (New York: Alan R. Liss), pp 315-359

26. Ruvinskii AO, Agul'nik SI, Agul'nik AI, Beliaev DK (1986) Chromosome segregation in mice heterozygous for Robertsonian translocation. II. Changes in the structure of homologs as a cause of abnormal disjunction in females. Genetika 10, 2505-2511

27. Sandell LL, Guan XJ, Ingram R, Tilghman SM (2003) Gatm, a creatine synthesis enzyme, is imprinted in mouse placenta. Proc Natl Acad Sci 100(8), 4622-4627

28. Tease C, Fisher G (1991) Two new X-autosome Robertsonian translocations in the mouse. Meiotic chromosome segregation in male hemizygotes and female heterozygotes. Genet Res 58, 115-121

29. Underkoffler LA, Mitchell LE, Localio AR, Marchegiani SM, Morabito J, et al. (2002) Molecular analysis of nondisjunction in mice heterozygous for a Robertsonian translocation. Genetics 161, 1219-1224

30. Underkoffler LA, Mitchell LE, Abdulali ZS, Collins JN, Oakey RJ (2005) Transmission ratio distortion in offspring of mouse heterozygous carriers of a (7.18) Robertsonian translocation. Genetics 169, 843-848

31. Wallace BMN, Searle JB, Everett CA (1992) Male meiosis and gametogenesis in wild house mice (mus musculus domesticus) from a chromosomal hybrid zone: comparison between "simple" Robertsonian heterozygotes and homozygotes. Cytogenet Cell Genet $61,211-220$

32. Warburton D (1997) Human female meiosis: New insights into an error-prone process. Am J Hum Genet $61,1-4$

33. Warburton D, Kinney A (1996) Chromosomal differences in susceptibility to meiotic aneuploidy. Environ Mol Mutagenesis 28, 237-247

34. Wroe SF, Kelsey G, Skinner JA, Bodle D, Ball ST, et al. (2000) An imprinted transcript, antisense to Nesp, adds complexity to the cluster of imprinted genes at the mouse Gnas locus. Proc Natl Acad Sci 97/7), 3342-3346 